\title{
Narcissism: Psychometrics and Social Behavior
}

\author{
Introduction to the Special Issue
}

\author{
Anthony D. Hermann ${ }^{1}$
}

Published online: 8 April 2016

(C) Springer Science+Business Media New York 2016

I am pleased to introduce our special issue entitled Narcissism: Psychometrics and Social Behavior. Few would argue that Western society is currently highly attuned to expressions of narcissism in popular and political culture. Self-help books that offer help in coping with narcissistic co-workers and significant others abound. Political leaders display narcissistic grandstanding with surprising regularity. Social media offer unprecedented opportunities for narcissistic self-expression. More systematic evidence also suggests that the personality of this generation is more narcissistic (Twenge, Konrath, Foster, Campbell, \& Bushman, 2008), less empathetic (Konrath, O’Brien, \& Hsing, 2011), more boastful (Twenge, Campbell, \& Gentile, 2012), and less trusting (Twenge, Campbell, \& Carter, 2014) than previous generations. Thus, the papers in this special issue provide a timely and engaging examination of how individual differences in narcissism can be better understood and measured and their impact on a wide variety of social behavior- fame seeking, volunteering, charitable donations, and prayer, just to name a few.

The mixed bag of characteristics and motivations that compose trait narcissism is clearly evident in this collection of studies. For example, Konrath, Ho, and Zarins present evidence that narcissists in some cases are more likely to engage in some prosocial behavior, especially those that are public in

Anthony D. Hermann

ahermann@bradley.edu; http://www.tonyhermann.com

1 Bradley University, Bradley Hall 89, 1501 W Bradley Ave, Peoria, IL 6162, USA nature, but are less likely to do so if the behavior is explicitly altruistic. Simpson, Hermann, Lehtman, and Fuller show that narcissists are willing to report feeling guilty and ashamed after committing a relationship transgression, but are less likely to turn to God in such situations, an outcome that could be perceived as adaptive or maladaptive depending on your view of such behavior. Foster, Shiverdecker, and Turner provide evidence that the Narcissistic Personality Inventory (NPI), the most widely used measure of trait narcissism, measures more psychologically healthy features on the lower end of the score continuum and more antagonistic features on the higher end.

The studies in this collection also contain a number of intriguing and surprising findings that deserve to be explored further. For example, Brunell and Davis found that the leadership/authority facet of trait narcissism to have the strongest relation to the desire to retaliate after unfair treatment, a finding that hints at the dark side of this more adaptive aspect of narcissism. Wallace, Sheiner, and Grotzinger find that narcissists are not more or less tolerant routine anti-social behaviors than non-narcissists, but they are more likely report engaging in such behaviors, a finding that points at the shifting standards that narcissists seem to have about their own behavior. Siedor, Maples-Keller, Miller, and Campbell found that although trait grandiose narcissism is strongly related to hypomanic personality overall, it was not related to its mood volatility facet, a finding that is a bit surprising given past research regarding narcissists' reactions to perceived transgressions. Southard and Zeigler-Hill found that the grandiosity subscale of the Pathological Narcissism Inventory was positively related to most aspects of fame-seeking, but it was unrelated to drive to achieve fame, a dimension positively correlated in previous research using the NPI, a more well-used measure of 
grandiose narcissism. Taken together these and other findings in our issue highlight the fascinating and multidimensional nature of narcissism and the value of empirical research on this popular topic.

In conclusion, I thank all the authors in this special issue for their conscientious efforts to produce well-designed studies, high-quality writing, and valuable mentoring to their students. I hope you enjoy reading these papers as much as I editing them.

\section{References}

Konrath, S. H., O’Brien, E. H., \& Hsing, C. (2011). Changes in dispositional empathy in American college students over time: A meta-analysis. Personality and Social Psychology Review, 15(2), 180-198. doi:10.1177/1088868310377395.

Twenge, J. M., Konrath, S., Foster, J. D., Campbell, W. K., \& Bushman, B. J. (2008). Egos inflating over time: A crosstemporal meta-analysis of the Narcissistic Personality Inventory. Journal of Personality, 76(4), 875-902. doi:10. 1111/j.1467-6494.2008.00507.x.

Twenge, J. M., Campbell, W. K., \& Gentile, B. (2012). Generational increases in agentic self-evaluations among American college students, 1966-2009. Self and Identity, 11(4), 409-427. doi:10. 1080/15298868.2011.576820.

Twenge, J. M., Campbell, W. K., \& Carter, N. T. (2014). Declines in trust in others and confidence in institutions among American adults and late adolescents, 1972-2012. Psychological Science, 25(10), 1914-1923. doi:10.1177/0956797614545133. 